\title{
Compósitos de Matriz Poliéster Reforçados por Fibras Curtas de Sisal
}

\author{
Kuruvilla Joseph, Eliton S. Medeiros e Laura H. Carvalho
}

Resumo: As propriedades tênseis de compósitos de matriz poliéster, reforçados por fibras curtas de sisal foram determinadas em função do comprimento, teor e orientação das fibras. Os resultados mostram que, para os compósitos investigados, a resistência à tração aumentou com o comprimento das fibras (5 - $45 \mathrm{~mm})$, atingiu um plateau $(45-55 \mathrm{~mm})$ e então decresceu $(55-75 \mathrm{~mm})$ enquanto os valores de módulo e de alongamento na ruptura não dependiam do comprimento das fibras. A resistência à tração dos compósitos aumentou com o teor de fibras até $50 \%$ em peso e, a partir daí, tendeu a decrescer. A resistência à tração de compósitos reforçados por fibras orientadas e testados longitudinalmente ao reforço foi 10 vezes maior do que a sua resistência transversal e 3 vezes maior do que a resistência de compósitos reforçados por fibras dispostas ao acaso.

Palavras-chave: Fibras de sisal, poliéster, compósitos, comprimento de fibras, orientação, propriedades mecânicas.

\section{Introdução}

$\mathrm{Na}$ última década tem havido um rápido desenvolvimento na área de compósitos reforçados por fibras vegetais. As fibras celulósicas possuem muitas características que tornam seu uso vantajoso como: baixo custo, baixa densidade, resistência específica e módulo elevados, não são abrasivas e, portanto, não desgastam os equipamentos de processo, não são tóxicas, podem ser facilmente modificadas por agentes químicos, são abundantes e provém de fontes renováveis. Suas propriedades mecânicas são comparáveis a de outros reforços comumente empregados. As fibras vegetais são bem mais baratas do que as fibras sintéticas e podem substitui-las em muitas aplicações onde o custo é fator mais importante do que a resistência. Fibras vegetais como juta, sisal, coco e abacaxi tem sido utilizadas como reforço em matrizes poliméricas ${ }^{[1-}$ ${ }^{2]}$. Dentre estas fibras, o uso do sisal é particularmente interessante já que seus compósitos possuem elevada resistência ao impacto além de moderada resistência à tração e em flexão quando comparados a compósitos reforçados por outras fibras vegetais $^{[3-4]}$. O sisal é uma das fibras vegetais mais extensamente utilizadas e é responsável por metade da produção total de fibras têxteis ${ }^{[5,6]}$. Barkakaty ${ }^{[5]}$ estudou os aspectos estruturais das fibras de sisal. Mukherjee e Satyanarayana ${ }^{[6]}$ descreveram a estrutura e propriedades das fibras de sisal. Satyanarayana e colaboradores ${ }^{[7]}$ reportaram que estas fibras podem ser utilizadas na manufatura de chapas planas e corrugadas que, além de resistentes e baratas são resistentes à chama. Há estudos sendo realizados

Kuruvilla Joseph, Dept. Chemistry, St. Berchman's College, Changanacherrry, Kerala, India.; Eliton S. de Medeiros e Laura H. Carvalho, DEMa, CCT, Universidade Federal da Paraíba, C.P. 10034, CEP:58109-970, Campina Grande, PB, email: laura@dema.ufpb.br 
visando melhoramentos no rendimento e qualidade das fibras produzidas bem como alterações em sua composição química para melhor adequa-las a determinadas aplicações ${ }^{[8]}$.

A incorporação de fibras de sisal a plásticos tem sido reportada por vários pesquisadore ${ }^{[9-15]}$. Joseph e colaboradores ${ }^{[9-12]}$ investigaram as propriedades mecânicas, reológicas, elétricas e viscoelásticas de compósitos de polietileno de baixa densidade reforçados por fibras curtas de sisal em função do processamento utilizado, teor, orientação e comprimento das fibras. Segundo os autores, a incorporação das fibras à matriz através da fusão (extrusão) leva à quebra excessiva das fibras, o que pode ser evitado através de uma mistura por solução. Os autores mostram que o processo de extrusão leva ao alinhamento das fibras curtas e que a resistência e módulo de compósitos extrusados, testados ao longo do eixo de alinhamento das fibras, foi significativamente maior (mais de 2 vezes) do que os de compósitos em que o reforço achava-se distribuído ao acaso. Kalaprasad e colaboradores ${ }^{[13]}$ reportaram a influência da adição de fibras curtas de vidro nas propriedades mecânicas de compósitos de polietileno de baixa densidade reforçado por sisal. Observaram que a adição de um pequeno teor $(3 \%$ em volume) de fibras de vidro levou a um aumento de mais de $80 \%$ na resistência longitudinal (fibras orientadas) do compósito. Observaram também que a tendência à absorção de água destes compósitos decresceu com a hibridização. Le Thi e colaboradores ${ }^{[14]}$ estudaram as propriedades mecânicas de compósitos polipropileno, preparados por extrusão reativa com anidrido maleico, reforçados por fibras de sisal. Reportaram que a enxertia das fibras, ocorrida durante a extrusão reativa do compósito, elevou a resistência ao impacto e a tensão à ruptura dos compósitos PP/sisal. Manikandan e colaboradores $^{[15]}$ reportaram o efeito do teor, comprimento, orientação e benzoetilação das fibras nas propriedades tênseis de compósitos PP/sisal. Segundo os autores, a benzoetilação das fibras melhora a adesão fibra-matriz e, por conseguinte, eleva significativamente a resistência dos compósitos cujas propriedades eram praticamente independentes do comprimento de fibras, se bem que, a resistência máxima na ruptura tenha apresentado um leve aumento para compósitos reforçados por fibras de $10 \mathrm{~mm}$

A incorporação de sisal como elemento de re- forço em termoplásticos tem sido reportada por vários autores ${ }^{[16-23]}$. Parmasivan e Adbulkalam ${ }^{[16]}$ investigaram a viabilidade do desenvolvimento de compósitos poliméricos de baixo custo reforçados por fibras de sisal pois estas fibras se prestam a vários processos de conformação de compósitos como por enrolamento ou laminação. Pavithran e colaboradores ${ }^{[3,19]}$ compararam as propriedades de impacto de compósitos poliéster-sisal reforçados por fibras de sisal alinhadas unidirecionalmente com as de compósitos de polietileno de ultra alto módulo (UHDMPE) reforçado por fibras de vidro. Observaram que os compósitos reforçados por sisal apresentam trabalho de fratura idêntico ao dos compósitos de polietileno de ultra alto módulo reforçados por fibra de vidro e que, se a densidade dos compósitos for considerada, a tenacidade dos compósitos reforçados por sisal é $25 \%$ inferior à dos compósitos reforçados por fibra de vidro. O elevado trabalho de fratura obtido para os compósitos reforçados por sisal , apesar destas fibras apresentarem baixos valores de resistência e módulo confirma os argumentos levantados pelos autores que a previsão das propriedades de impacto de compósitos reforçados por fibras vegetais não será válida se a contribuição da estrutura helicoidal microfibrilar da fibras vegetal não for considerada. É fato geralmente aceito que a tenacidade de compósitos reforçados por fibras depende primordialmente do comportamento tensão-deformação da fibra. Fibras resistentes com alta deformação de ruptura elevam o trabalho de fratura dos compósitos ${ }^{[20]}$. Bisanda e Ansel[ ${ }^{[23]}$ avaliaram as propriedades de compósitos epoxi/sisal e reportaram que a incorporação do sisal à matriz epoxi gera produtos rígidos e resistentes.

O presente trabalho trata da otimização das propriedades de compósitos de matriz poliéster insaturado em função do comprimento e teor de fibras de sisal empregados. A influência do comprimento e teor das fibras empregadas nas propriedades dos compósitos foi analisada. As propriedades destes compósitos foram comparadas a de outros semelhantes reforçados por fibras de sisal longas e alinhadas unidirecionalmente.

\section{Experimental}

As fibras de sisal, fornecidas pela Brascorda S.A, foram lavadas com água, secas ao ar e corta- 
das nas dimensões desejadas antes de serem utilizadas como reforço em compósitos de matriz poliéster fornecida pela Resana S.A. de código Resapol 10-225. Os compósitos foram moldados por compressão na forma de placas de 200x150x2mm. As fibras de sisal, em quantidade e tamanho pré-determinados foram espalhadas uniformemente no molde e a resina, catalisada por MEK a $1 \%$, vertida sobre as fibras e pressionada com o auxílio de um bloco de madeira para garantir o molhamento das fibras e remoção de bolhas. O molde foi fechado e mantido sob pressão de ( 8 ton de força) por 8 horas. As placas foram pós-curadas por $8 \mathrm{~h}$ a $80^{\circ} \mathrm{C}$ e corpos de prova confeccionados a partir destas placas. Os testes foram conduzidos segundo as normas ASTM D-3039 em máquina universal de ensaios Testometric Micro 350, operando a $5 \mathrm{~mm} / \mathrm{min}$. O módulo e alongamento na ruptura foram calculados a partir das curvas tensão-deformação e os valores médios de pelo menos seis amostras foram reportados.

\section{Resultados e Discussão}

A Tabela 1 mostra o efeito do comprimento das fibras nas propriedades tênseis de compósitos poliéster/fibras curtas de sisal, distribuidas ao acaso, com um teor de fibras de $30 \%$ em peso.

Fica evidenciado que a resistência dos compósitos aumenta com o comprimento de fibras de 5 a $45 \mathrm{~mm}$, tendendo a manter-se neste mesmo patamar e, finalmente, decrescer para comprimentos maiores. Este nivelamento e posterior decréscimo da resistência dos compósitos reforçados por fibras mais longas do que $45 \mathrm{~mm}$ é atribuido ao emaranhamento

Tabela 1. Efeito do comprimento das fibras nas propriedades de compósitos poliéster/fibras curtas de sisal

\begin{tabular}{cccc}
\hline $\begin{array}{c}\text { Fibras } \\
(\mathbf{m m})\end{array}$ & $\begin{array}{c}\text { Resist. tração } \\
\text { na ruptura } \\
\text { (Mpa) }\end{array}$ & $\begin{array}{c}\text { Módulo elástico } \\
\text { (Gpa) }\end{array}$ & $\begin{array}{c}\text { Alongamento } \\
\text { na ruptura } \\
(\%)\end{array}$ \\
\hline 5 & $26,68 \pm 0,67$ & $2,13 \pm 0,08$ & $4,770 \pm 0,55$ \\
15 & $37,25 \pm 2,01$ & $2,20 \pm 0,41$ & $5,074 \pm 0,57$ \\
25 & $40,84 \pm 0,88$ & $2,19 \pm 0,13$ & $5,841 \pm 0,88$ \\
35 & $43,36 \pm 1,86$ & $2,13 \pm 0,01$ & $6,119 \pm 0,25$ \\
45 & $44,16 \pm 1,67$ & $2,19 \pm 0,14$ & $6,166 \pm 0,03$ \\
55 & $44,49 \pm 1,97$ & $2,09 \pm 0,04$ & $6,152 \pm 0,19$ \\
65 & $40,96 \pm 1,89$ & $2,01 \pm 0,06$ & $5,199 \pm 0,37$ \\
75 & $37,81 \pm 1,58$ & $1,94 \pm 0,08$ & $5,429 \pm 0,59$ \\
\hline
\end{tabular}

das fibras que ocorre a comprimentos maiores. $\mathrm{O}$ módulo e alongamento na ruptura dos compósitos, por sua vez, não foram alterados significativamente pelo comprimento das fibras de reforço. A resistência e módulo de compósitos reforçados por fibras curtas dependem principalmente da eficiência de transferência de tensões da matriz às fibras. A eficiência desta transferência de tensões, por sua vez, depende do comprimento das fibras e da magnitude das interações na interface fibra-matriz.

Em compósitos reforçados por fibras curtas há um comprimento crítico de fibras necessário para que a resistência máxima (transferência de tensões) possa ser alcançada. A razão de aspecto crítica depende da fração volumétrica das fibras e também da razão entre os módulos das fibras e da matriz(24). A baixo carregamento (baixa fração volumétrica de reforço) as fibras influenciam pouco a resistência do compósito que é dominada pela matriz. Acima de um volume crítico de fibras, a resistência dos compósitos aumenta com o teor de fibras. Em geral, quanto maior a razão de aspecto, menor é este volume crítico. A teores de fibras relativamente baixos, a razão de aspecto crítica permanece praticamente constante; decrescendo abruptamente a elevados teores de fibras(25). Se o comprimento da fibra empregada como reforço for inferior ao comprimento crítico, a fibra irá ser sacada da matriz (pull-out) e o compósito falhará a baixas tensões. Quando o comprimento das fibras é maior do que o comprimento crítico, a transferência de tensões torna-se eficiente e haverá rompimento das fibras, de modo que compósitos de elevada resistência mecânica são obtidos.

Os resultados, portanto, indicam que há um comprimento ótimo de fibras entre 35 e $45 \mathrm{~mm}$, onde um máximo na resistência dos compósitos pode ser obtido. $\mathrm{O}$ motivo pelo qual a resistência não segue crescendo com o comprimento das fibras é atribuido a um emaranhamento e enrolamento mais severo do reforço a comprimentos maiores, reduzindo o seu comprimento efetivo. No caso de compósitos reforçados por fibras mais curtas do que este comprimento crítico, as fibras são sacadas da matriz durante o esforço mecânico e o compósito falha sob tensões relativamente baixas.

A Tabela 2 ilustra o efeito do teor de fibras nas propriedades mecânicas de compósitos poliéster/ sisal reforçados por fibras de $35 \mathrm{~mm}$ de comprimento distribuidas ao acaso. 
Tabela 2. Efeito do teor de fibras nas propriedades de compósitos poliéster/sisal de $35 \mathrm{~mm}$ de comprimento

\begin{tabular}{cccc}
\hline $\begin{array}{c}\text { Teor de fibras } \\
(\%)\end{array}$ & $\begin{array}{c}\text { Resist. tração } \\
\text { na ruptura } \\
(\mathbf{M p a})\end{array}$ & $\begin{array}{c}\text { Módulo elástico } \\
(\mathbf{G p a})\end{array}$ & $\begin{array}{c}\text { Alongamento } \\
\text { na ruptura } \\
(\%)\end{array}$ \\
\hline 0 & $34,8 \pm 2,51$ & $1,36 \pm 0,08$ & $2,07 \pm 0,13$ \\
10 & $23,30 \pm 1,40$ & $1,64 \pm 0,07$ & $3,31 \pm 0,88$ \\
20 & $31,83 \pm 1,80$ & $1,95 \pm 0,53$ & $5,15 \pm 0,53$ \\
30 & $43,36 \pm 1,85$ & $2,13 \pm 0,10$ & $6,12 \pm 0,25$ \\
40 & $48,18 \pm 1,92$ & $1,91 \pm 0,9$ & $7,20 \pm 0,31$ \\
50 & $59,74 \pm 1,61$ & $1,81 \pm 0,03$ & $7,80 \pm 0,30$ \\
60 & 55,13 & $1,72 \pm 0,10$ & $7,76 \pm 0,14$ \\
\hline
\end{tabular}

Os resultados mostram que a resistência dos compósitos é menor do que a da matriz a carregamentos de até $20 \%$ em peso de fibras. Aumentos subsequentes no teor de fibras (20 a $50 \%$ em peso) levam a aumentos consideráveis (57\% maior do que a resistência da matriz) na resistência dos compósitos, porém, para carregamentos maiores do que $50 \%$ a tendência se inverte. $\mathrm{O}$ fato da resistência dos compósitos, a baixos teores de fibras, ser inferior à da matriz é atribuido ao carregamento ineficiente, de modo que as fibras agem como defeitos e fragilizam a matriz, reduzindo a resistência mecânica do compósito. A baixos carregamentos, a matriz não é ancorada suficientemente pelas fibras e deformações locais elevadas ocorrem na matriz a baixas tensões, o que faz com que a ligação fibra-matriz se rompa e a matriz seja diluida por fibras não reforçantes e sacadas. A medida em que o teor de fibras aumenta, as tensões passam a ser mais uniformemente distribuidas e a resistência do compósito aumenta. Os resultados indicam que a carregamentos superiores a $50 \%$ em peso, a resistência dos compósitos tende a decrescer. Isto muito provavelmente se deve à maior interação entre as fibras, aumentando seu diâmetro efetivo e reduzindo a razão de aspecto a carregamentos muito elevados.

$\mathrm{O}$ efeito do teor de fibras nos módulos dos compósitos seguiu a mesma tendência apresentada para a resistência, ou seja, aumento seguido de decréscimo a teores mais elevados. Um máximo nesta propriedade foi obtido a um carregamento de $30 \%$ de fibras onde a rigidez do compósito é cerca de $70 \%$ maior do que a da matriz. O alongamento na ruptura aumenta com o teor de fibras, o que é tido como indicativo de uma redução na rigidez da matriz com a adição das fibras de sisal,
Tabela 3. Efeito da orientação das fibras nas propriedades de compósitos poliéster/sisal

\begin{tabular}{cccc}
\hline $\begin{array}{c}\text { Tipo de } \\
\text { compósito }\end{array}$ & $\begin{array}{c}\text { Resist. tração } \\
\text { na Ruptura } \\
\text { (Mpa) }\end{array}$ & $\begin{array}{c}\text { Módulo elástico } \\
\text { Gpa) }\end{array}$ & $\begin{array}{c}\text { Alongamento } \\
\text { na Ruptura } \\
(\%)\end{array}$ \\
\hline Tipo I & $43,36 \pm 1,85$ & $2,13 \pm 0,01$ & $6,12 \pm 0,25$ \\
Tipo II & $115,82 \pm 3,01$ & $3,07 \pm 0,09$ & $6,28 \pm 0,27$ \\
tipo III & $11,59 \pm 1,33$ & $0,91 \pm 0,06$ & $1,02 \pm 0,22$ \\
\hline
\end{tabular}

onde: Tipo I - compósito com fibras de $35 \mathrm{~mm}$ distribuidas ao acaso; Tipo II - compósito reforçado por fibras longas orientadas, testados na direção longitudinal e Tipo III - compósito reforçado por fibras longas orientadas e testado na direção transversal ao reforço. Em todos os casos o teor de fibras foi de $30 \%$ em peso.

ou seja, na medida em que o alongamento e a resistência dos compósitos aumenta com o teor de fibras, pode-se esperar um aumento nas propriedades de impacto do compósito com o teor de fibras.

A Tabela 3 compara as propriedades de compósitos reforçados por fibras dispostas ao acaso com a de compósitos unidirecionais reforçados por fibras longas. Em ambos os casos, o teor de fibras foi mantido em $30 \%$ em peso.

Os resultados indicam que a resistência à tração de compósitos unidirecionais testados longitudinalmente ao reforço, tem resistência 300\% maior do que a de compósitos com fibras dispostas ao acaso. A orientação das fibras exerce papel fundamental na resistência de materiais compósitos e dois casos extremos são possíveis: a) alinhamento paralelo das fibras ao longo do eixo longitudinal das fibras numa só direção e b) disposição ao acaso. Compósitos longitudinalmente orientados são anisotrópicos por natureza e o máximo na resistência é obtido quando o material é testado na direção do alinhamento das fibras. Na direção transversal ao alinhamento das fibras o reforço é virtualmente inexistente e, portanto, o material falha a tensões muito baixas. Compósitos reforçados por fibras dispostas ao acaso apresentam propriedades intermediárias às exibidas por compósitos unidirecionais. A Figura 1 ilustra o aspecto de compósitos reforçados por fibras distribuidas ao acaso e fibras longas alinhadas unidirecionalmente. Como mencionado anteriormente, as propriedades de compósitos unidirecionais testados longitudinalmente ao reforço são bastante superiores às dos mesmos compósitos testados transversalmente às fibras ou de compósitos reforçados por fibras distribuidas ao acaso. Quando o teste é realizado transversalmente ao 


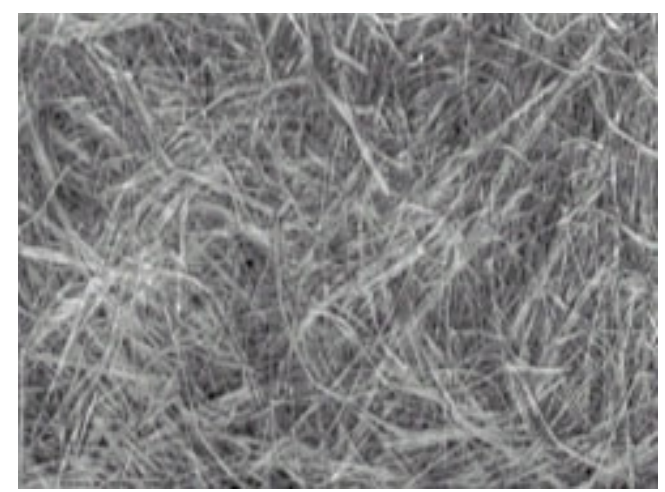

(a)

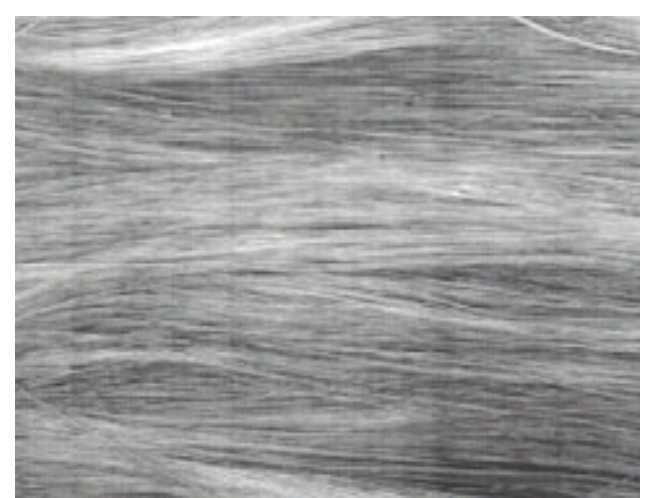

(b)

Figura 1. Aspecto dos compósitos com fibras orientadas aleatoriamente (a) ou unidiriecionalmente (b).

reforço as fibras não são capazes de transferir tensões e aumentar a resistência da matriz. Como esperado, as propriedades de compósitos reforçados por fibras distribuidas ao acaso apresentaram valores intermediários a estes dois extremos. Fica evidenciado que, mesmo um pequeno desalinhamentos das fibras em compósitos unidirecionais testados longitudinalmente ao reforço, pode levar a um decréscimo dramático na resistência e módulo destes compósitos.

\section{Conclusões}

A influência do teor, comprimento e orientação das fibras nas propriedades tênseis de compósitos poliéster/sisal reforçados por fibras curtas de sisal dispostas ao acaso foi determinada. Os resultados indicam que as propriedades aumentaram com o comprimento das fibras e que o comprimento crítico situa-se na faixa de $35-45 \mathrm{~mm}$. Fibras mais longas emaranham e curvam-se severamente, reduzindo seu comprimento efetivo. O módulo e o alongamento na ruptura dos compósitos praticamente não foi afetado pelo comprimento das fibras. A resistência dos compósitos aumenta com teores de fibra entre 20 e $50 \%$. A teores menores do que $20 \%$ o carregamento é ineficiente e, acima de $50 \%$ em peso há excessiva interação entre as fibras e as propriedades dos compósitos diminuem. Um compósito com resistência à tração $57 \%$ superior à da matriz pode ser obtido a um carregamento de $50 \%$ em peso de fibras. No que se refere à orientação das fibras, a resistência de compósitos reforçados por fibras longas unidirecionais e testados longitudinalmente ao reforço foi 10 vezes maior do que quando testados transversalmente ao reforço e 3 vezes maior do que a de compósitos semelhantes reforçados por fibras distribuidas ao acaso. Os resultados demonstram que compósitos de matriz poliéster reforçados por fibras de sisal apresentando bom conjunto de propriedades mecânicas, adequados a várias aplicações puderam ser obtidos.

\section{Agradecimentos}

Os autores agradecem à Resana S.A. e Brascorda S.A p pela doação dos materiais e ao $\mathrm{CNPq}$ pelas bolsas de pesquisa concedidas.

\section{Referências Bibliográficas}

1. McLaughlin, E. C. - J Mater - Sci., 15, 886 (1980).

2. Prasad, S. V.; Pavithran, C. and Rohatgi, P. K. J. Mater. Sci., 12, 1443 (1983).

3. Pavithran, C.; Mukherjee, P. S. Brahmakumar, M.and Damodaran, A. D. - J. Mater.Sci.Lett., 7, 825 (1988).

4. Pavithran, C.; Mukherjee, P. S. and Brahmakumar, M. and Damodaran, A. D. - J. Mater.Sci., 26,455 (1991).

5. Barkakaty, B. C. - J. Appl. Polym. Sci., 20, 2921(1976).

6. Mukherjee, P. S. and Satyanarayana, K. G. J.Mater.Sci., 21, 51 (1986).

7. Satyanarayana, K. G.; Kulkarni, A.G. and Rohatgi, P. K. - J. Scif. Ind. Res., 40, 222 (1981). 
8. Dhyani, K. C. and Geo Paul, N. - Jute Bull., 37, 1 (1974).

9. Joseph K.; Sabu, T. and Pavithran, C. - J. Reinf. Plast. and Comp., 12, 139 (1993b).

10. Joseph K.; Pavithran C.; Sabu T., Kuriakose, B. and Premalatha, C. K. - Plast. Rubb. Comp. Proc. and Appl., 21, 237 (1994).

11. Joseph K.; Sabu, T. and Pavithran, C. - Polymer, 37, 23 (1996b).

12. Joseph, K.; Sabu, T. and Pavithran, C. - Comp. Sci., and Techno., 53, 99 (1995b).

13. Kalaprasad G.; Kuruvilla, J. and Sabu T. - J. of Comp. Mater., 31, 5 (1997b).

14. LeTHI T. T.; Gauthier and Chabert B. - J. of Mater. Sci.- Pure and Appl. Chem., Vol. A 33(12), pp-1997-2004 (1996).

15. Manikandan Nair K. C., Diwan S. M. and Sabu T. - J. of Appl. Polym. Sci., Vol. 60, pp-1483-1497 (1996).

16. Paramasivan, V. and A.P.J. Abdulkalam, Fibre. Sci. Tech. 7, 85 (1974).
17. Mauersberger, H. R. (Ed.), "Textile Fibres", John Wiley \& Sons. Inc., New York, (1974).

18. Norton, R.R. (Ed.), "Composite Materials: Engineering Applications of Composites", Vol. 3, Academic Press. Inc., New York, (1974).

19. Pavithran, C.; Mukherjee, P. S.; Brahmakumar, M. and Damodaran, A. D. - J. Mater. Sci. Lett., 7, 882 (1987).

20. Fujii, T. - J. Jpn. Soc. Comp. Mater, 1, 35 (1975).

21. Gopal, M.; Bhaduri, S. K. and Rao, K. P. - Ind., J. Text. Res., 10, 68 (1985).

22. Samal, B. B.; Sahu, S.; Chinara, B. B.; Nanda, S.; Otta, P. K.; Mohapatro, L. M.; Mohanty, T. R.; Ray, A. R. and Singh, K. C. - J. Appl. Polym,. Sci. 26, 3159 (1988).

23. Bisanda, E. T. N. and Ansell, M. P. - Comp. Sci. Technol., 41, 165 (1991).

24. Termonia, Y. - J. Mater. Sci., 22, 504 (1989).

25. Termonia, Y. - J. Mater. Sci., 25, 4644 (1990). 